\title{
The probability that an operator is nilpotent
}

\author{
Tom Leinster*
}

\begin{abstract}
Choose a random linear operator on a vector space of finite cardinality $N$ : then the probability that it is nilpotent is $1 / N$. This is a linear analogue of the fact that for a random self-map of a set of cardinality $N$, the probability that some iterate is constant is $1 / N$. The first result is due to Fine, Herstein and Hall, and the second is essentially Cayley's tree formula. We give a new proof of the result on nilpotents, analogous to Joyal's beautiful proof of Cayley's formula. It uses only general linear algebra and avoids calculation entirely.
\end{abstract}

\section{Introduction}

This note is about the theorem that on a finite-dimensional vector space $X$ over a finite field, the probability of a randomly-chosen linear operator being nilpotent is 1 over the number of elements of $X$. If the field has order $q$ and $\operatorname{dim} X=n$ then there are $q^{n}$ elements of $X$ and $q^{n^{2}}$ operators on $X$, so an equivalent result is that $X$ admits $q^{n(n-1)}$ nilpotents.

This theorem was first published in 1958 by Fine and Herstein [3], who gave a calculation-heavy proof using the theory of partitions. Subsequently, others found proofs needing less calculation: first Gerstenhaber [4] in 1961 (avoiding partitions), then Kaplansky [7] in 1990 (with an inclusion-exclusion argument), then Crabb [2] in 2006 (imitating the proof of Cayley's formula by Prüfer codes), then Brouwer, Gow and Sheekey [1] in 2014 (with a very efficient proof using the Fitting decomposition and $q$-binomial coefficients). Brouwer, Gow and Sheekey also mention unpublished 1955 lecture notes of Philip Hall, stating that he gave two proofs, 'one involving a form of Möbius inversion, the other exploiting the theory of partitions' ([1], Section 2.1).

This note gives a new proof requiring no calculation or manipulation of algebraic expressions at all - at least, granted some standard linear algebra. It is analogous to Joyal's beautiful proof of Cayley's formula ([6], p. 16), in the following sense.

Cayley's formula states that for a finite set $X$ with $N>0$ elements, the number of (unrooted) trees with vertex-set $X$ is $N^{N-2}$. A rooted tree is a tree together with a choice of vertex, called the root; there are $N^{N-1}$ of these. They can be identified with the functions $T: X \rightarrow X$ that are eventually constant, meaning that $T^{k}=T \circ \cdots \circ T$ is constant for some $k \geq 0$. Indeed, if we orient the edges of a rooted tree towards the root, the resulting diagram depicts an eventually constant function, with the root $z$ as the eventual constant value and an edge from $x$ to $T(x)$ for each $x \neq z$. Hence there are $N^{N-1}$ eventually constant functions $X \rightarrow X$. Equivalently, the probability of a random function $X \rightarrow X$ being eventually constant is $N^{N-1} / N^{N}=1 / N$.

When $X$ is a vector space, the eventually constant linear maps $T: X \rightarrow X$ are exactly the nilpotent operators (those satisfying $T^{k}=0$ for some $k$ ). So

*School of Mathematics, University of Edinburgh, Scotland; Tom.Leinster@ed.ac.uk 


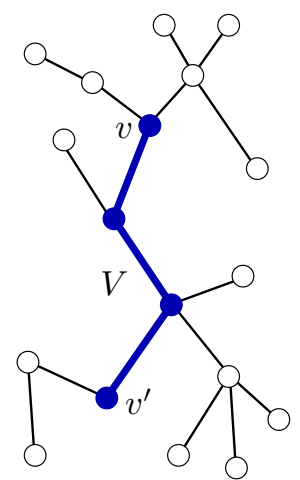

(a)

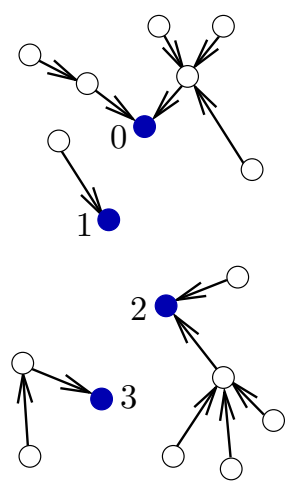

(b)

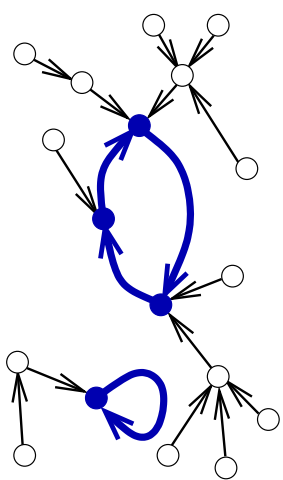

(c)

Figure 1: Joyal's proof of Cayley's formula

Cayley's formula and the theorem on nilpotents both state, in different contexts, the probability that a random self-map is eventually constant.

Joyal proved Cayley's formula by constructing a bijection

$$
\{\text { trees on } X\} \times X \times X \cong\{\text { functions } X \rightarrow X\}
$$

for any nonempty finite set $X$. His construction depends on an arbitrary choice, for each $V \subseteq X$, of a bijection between the total orders on $V$ and the permutations of $V$. It runs as follows. An element of the left-hand side of (1) is a tree with chosen vertices $v$ and $v^{\prime}$. There is a unique path from $v$ to $v^{\prime}$, and the set $V$ of vertices along that path is naturally ordered (Fig. 1(a)). Thus, a tree on $X$ with two distinguished vertices amounts to a totally ordered subset $V$ of $X$ together with a family of rooted trees partitioning $X$, the roots being the elements of $V$ (Fig. 1(b)). As before, we can harmlessly orient the edges of these trees towards their roots.

We can equivalently replace the total order on $V$ by the corresponding permutation. This produces a diagram as in Fig. 1(c) (where the permutation shown is necessarily arbitrary). But such a diagram is simply the graph of a function $X \rightarrow X$, with $V$ as its set of periodic points. This completes the proof.

Our proof of the formula for nilpotents follows a similar pattern. For example, Joyal's argument uses the decomposition of an endomorphism of a finite set into a permutation and some eventually constant functions, and our argument uses the decomposition of an endomorphism of a finite-dimensional vector space into an automorphism and a nilpotent. However, the translation to the linear context is not entirely mechanical. An important difference is that whereas a subset of a set has only one complement, a subspace of a vector space has many complementary subspaces.

One feature of Joyal's proof is that the total orders and permutations of a finite set $V$ are in non-canonical bijection. This situation can be understood through Joyal's theory of species [6], or by noting that we have a torsor: a nonempty set $S$ acted on by a group $G$ in such a way that for each $s, s^{\prime} \in S$, there is a unique $g \in G$ satisfying $g s=s^{\prime}$. Every element $s_{0} \in S$ defines a bijection $G \cong S$ by $g \leftrightarrow g s_{0}$, but in general there is no canonical bijection $G \cong S$ : for which element of $S$ would correspond to the identity element of $G$ ? In the case at hand, $G$ is the group of permutations of $V$ acting on the set of total orders on $V$. We will use an analogous torsor in the linear context. 


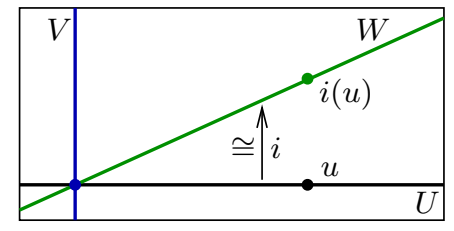

Figure 2: The canonical isomorphism $i$ between complements $U$ and $W$ of $V$

\section{Background linear algebra}

Throughout, all vector spaces are over an arbitrary field, not necessarily finite.

Subspaces $U$ and $V$ of a vector space $X$ are complementary if $U \cap V=\{0\}$ and $U+V=X$; we also say that $U$ is a complement of $V$. Although a subspace generally has many complements, any two are canonically isomorphic. Indeed, let $U$ and $W$ be complements of $V \subseteq X$; then there is an isomorphism $i: U \rightarrow W$ defined by taking $i(u)$ to be the unique element of $W$ such that $i(u)-u \in V$ (Fig. 2).

For a linear map $f: U \rightarrow V$ between vector spaces $U$ and $V$, its graph

$$
W_{f}=\{(u, v) \in U \oplus V: f(u)=v\}
$$

is a complement of $V$ in $U \oplus V$. Moreover, for any complement $W$ of $V$ in $U \oplus V$, there is a unique linear map $f: U \rightarrow V$ such that $W_{f}=W$ : in the notation of the previous paragraph, $f(u)=i(u)-u$. Hence:

Lemma 1 For vector spaces $U$ and $V$, there is a canonical bijection

$$
\{\text { linear maps } U \rightarrow V\} \cong\{\text { complements of } V \text { in } U \oplus V\} \text {. }
$$

Now we turn to nilpotents. Our first lemma is a straightforward induction.

Lemma 2 Let $T$ be a nilpotent operator on a vector space $X$, and let $v \in X$. Let $k \geq 0$ be least such that $T^{k}(v)=0$. Then $v, T(v), \ldots, T^{k-1}(v)$ are linearly independent.

Lemma 3 Let $U$ and $V$ be vector spaces and let $T$ be a linear operator on $U \oplus V$ such that $T V \subseteq V$. Write $\left(\begin{array}{cc}T_{U U} & 0 \\ T_{U V} & T_{V V}\end{array}\right)$ for the block decomposition of $T$. Then

$T$ is nilpotent $\Longleftrightarrow T_{U U}$ and $T_{V V}$ are nilpotent.

Proof For $u \in U$ and $k \geq 0$, the $U$-component of $T^{k}(u, 0)$ is $T_{U U}^{k}(u)$. Hence if $T^{k}=0$ then $T_{U U}^{k}=0$, and similarly $T_{V V}^{k}=0$. Conversely, suppose that $T_{U U}^{\ell}=0$ and $T_{V V}^{m}=0$. Then $T^{\ell}(U \oplus V) \subseteq V$, so $T^{\ell+m}(U \oplus V) \subseteq T^{m} V=\{0\}$.

An automorphism of a vector space is an invertible operator. Every operator decomposes uniquely as the direct sum of a nilpotent and an automorphism:

Lemma 4 (Fitting) Let $Q$ be a linear operator on a finite-dimensional vector space. Then there is a unique pair $(W, V)$ of complementary $Q$-invariant subspaces such that $Q$ is nilpotent on $W$ and an automorphism of $V$.

Proof See standard texts such as Jacobson [5] (Section 3.4). Explicitly, $W=$ $\bigcup_{i \geq 0} \operatorname{ker}\left(Q^{i}\right)$ and $V=\bigcap_{i \geq 0} \operatorname{im}\left(Q^{i}\right)$.

Finally, let $X$ be a finite-dimensional vector space. The set of ordered bases $\left(x_{1}, \ldots, x_{n}\right)$ of $X$ is a torsor (in the sense of the Introduction) over the automorphism group $\operatorname{Aut}(X)$, so there is a bijection between the set of ordered bases and $\operatorname{Aut}(X)$. 


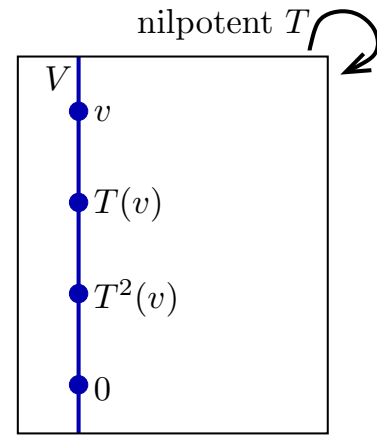

(a)

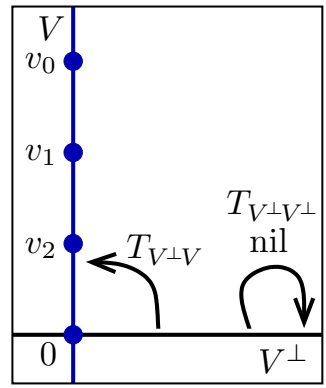

(b)

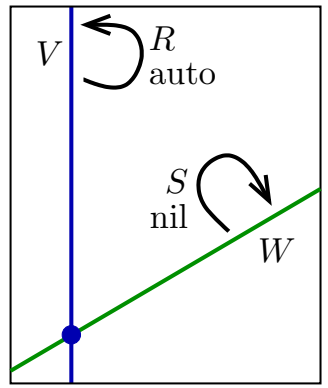

(c)

Figure 3: Schematic diagrams of the proof of Theorem 5

\section{The proof}

Theorem 5 Let $X$ be a finite-dimensional vector space over any field. Then there is a bijection

$$
\operatorname{Nil}(X) \times X \cong \operatorname{Lin}(X),
$$

where $\operatorname{Nil}(X)$ is the set of nilpotent operators on $X$ and $\operatorname{Lin}(X)$ is the set of all linear operators on $X$.

Proof For each subspace $V$ of $X$, choose a bijection between the ordered bases of $V$ and the automorphisms of $V$, and choose a complementary subspace $V^{\perp}$.

Let $(T, v) \in \operatorname{Nil}(X) \times X$. Write $V=\operatorname{span}\left\{T^{i}(v): i \geq 0\right\}$ (Fig. 3(a)). Let $k \geq 0$ be least such that $T^{k}(v)=0$, and put

$$
\mathbf{v}=\left(v_{0}, v_{1}, \ldots, v_{k-1}\right)=\left(v, T(v), \ldots, T^{k-1}(v)\right) .
$$

By Lemma 2, $\mathbf{v}$ is an ordered basis of $V$. Evidently $T V \subseteq V$, and the action of $T$ on $V$ is completely determined by $\mathbf{v}$ : it is the nilpotent $v_{0} \mapsto \cdots \mapsto v_{k-1} \mapsto 0$. Also, $v$ is determined by $\mathbf{v}$, since $v=0$ if $k=0$ and $v=v_{0}$ otherwise. The restriction $\left.T\right|_{V^{\perp}}: V^{\perp} \rightarrow X=V \oplus V^{\perp}$ decomposes as a linear map $T_{V^{\perp} V}: V^{\perp} \rightarrow V$ and a linear operator $T_{V^{\perp} V^{\perp}}$ on $V^{\perp}$. Hence by Lemma 3, to give a pair $(T, v)$ is equivalent to giving a linear subspace $V$ equipped with an ordered basis, a linear map $V^{\perp} \rightarrow V$, and a nilpotent on $V^{\perp}$ (Fig. 3(b)).

By Lemma 1, the linear maps $V^{\perp} \rightarrow V$ are in bijection with the subspaces $W$ of $X$ complementary to $V$. Given such a $W$, there is a canonical isomorphism $V^{\perp} \rightarrow W$ (as in Section 2), so we can equivalently replace the nilpotent $T_{V^{\perp} V^{\perp}}$ on $V^{\perp}$ by a nilpotent $S$ on $W$. Hence to give a pair $(T, v)$ is equivalent to giving a pair $(V, W)$ of complementary subspaces together with an ordered basis of $V$ and a nilpotent on $W$.

Now using the bijection chosen at the start of the proof, we can equivalently replace the ordered basis of $V$ by an automorphism $R$ of $V$ (Fig. 3(c)). Thus, we now have a pair $(V, W)$ of complementary subspaces of $X$, an automorphism of $V$, and a nilpotent on $W$. And by Lemma 4, to give such data is exactly to give a linear operator on $X$.

It follows that on a vector space with finite cardinality $N$, an operator chosen uniformly at random has probability $1 / N$ of being nilpotent. 
Remarks In both Joyal's proof of Cayley's formula and our proof of Theorem 5, the amount of arbitrary choice can be reduced. In the Cayley case, it suffices to choose a single total order on $X$ : for this induces a total order on each subset $V$, hence, via the torsor argument of the Introduction, a bijection between orders on and permutations of $V$. In the linear case, it suffices to choose a single ordered basis of $X$. The matrix echelon algorithm then produces an ordered basis of each subspace $V$, hence (by the torsor argument) a bijection between ordered bases and automorphisms of $V$. The Steinitz exchange algorithm produces a complement $V^{\perp}$ of each subspace $V$.

The proof of Theorem 5 establishes slightly more than is stated. For a nilpotent $T$ and a vector $v$, let $\operatorname{deg}_{v}(T)$ denote the least $k \geq 0$ such that $T^{k}(v)=0$. Then the proof shows that for all integers $k$,

$$
\left\{(T, v) \in \operatorname{Nil}(X) \times X: \operatorname{deg}_{v}(T)=k\right\} \cong\left\{Q \in \operatorname{Lin}(X): \operatorname{dim}\left(\bigcap_{i \geq 0} \operatorname{im} Q^{i}\right)=k\right\}
$$

Acknowledgements This work was supported by a Leverhulme Trust Research Fellowship. I thank Joachim Kock and Darij Grinberg for their comments.

\section{References}

[1] A. E. Brouwer, R. Gow, and J. Sheekey. Counting symmetric nilpotent matrices. The Electronic Journal of Combinatorics, 21(2):P2.4, 2014.

[2] M. C. Crabb. Counting nilpotent endomorphisms. Finite Fields and Their Applications, 12(1):151-154, 2006.

[3] N. J. Fine and I. N. Herstein. The probability that a matrix be nilpotent. Illinois Journal of Mathematics, 2(4A):499-504, 1958.

[4] M. Gerstenhaber. On the number of nilpotent matrices with coefficients in a finite field. Illinois Journal of Mathematics, 5(2):330-333, 1961.

[5] N. Jacobson. Basic Algebra II. W. H. Freeman and Company, San Francisco, 1980.

[6] A. Joyal. Une théorie combinatoire des séries formelles. Advances in Mathematics, 42(1):1-82, 1981.

[7] I. Kaplansky. Nilpotent elements in Lie algebras. Journal of Algebra, 133(2):467-471, 1990. 